EPJ Web of Conferences 73, 04010 (2014)

DOI: $10.1051 /$ epjconf/20147304010

(C) Owned by the authors, published by EDP Sciences, 2014

\title{
Latest results from FROST at Jefferson Lab
}

\author{
B.G. Ritchie ${ }^{\mathrm{a}}$ for the CLAS Collaboration
}

Arizona State University, USA

\begin{abstract}
The spectrum of broad and overlapping nucleon excitations can be greatly clarified by use of a polarized photon beam incident on a polarized target in meson photoproduction experiments. At Jefferson Lab, a program of such measurements has made use of the Jefferson Lab FROzen Spin Target (FROST). An overview of preliminary results are presented.
\end{abstract}

\section{Introduction}

As a composite system, the nucleon has a specific spectrum of excitations - the nucleon resonances. The details of this spectrum provide essential clues as to how this strongly-interacting three-quark system is constructed. Much effort has been expended using a variety of experimental probes to tease apart the excitations. From the theoretical side, many models of the nucleon have been developed to help understand the spectrum and to help guide experiments aimed at clarifying the properties of those excitations. However, most models predict more resonances than have been observed, the so-called "missing resonance" problem. Since nucleon excitations frequently overlap in energy due to their broad intrinsic widths (from about 50 to $300 \mathrm{MeV}$ ), it is not surprising that nearly half the states suggested by analyses of the existing data have only fair or poor evidence [1]. To advance our knowledge, new probes and observables are being explored to disentangle the spectrum.

The meson photoproduction reaction is a particularly useful tool for elucidating the details of the nucleon resonance spectrum. The observables for that process can be expressed conveniently and completely in terms of helicity observables (for example, Ref. [2]), which, in turn, can be compared to predictions for those amplitudes by nucleon models. A new generation of experiments is underway, incorporating polarized photon beams, polarized nucleon targets, and/or measurements of the polarization of final state particles, which provides better access to determining these helicity observables. Observables in which only the orientation of the target nucleon, the incoming photon, or the recoil particle is determined are called single-spin observables; these are the beam and target asymmetries $\Sigma$ and $T$, and the recoil polarization $P$. Double-spin observables have two of the three orientations (beam, target, recoil) determined; for all possible pairs within the set of orientations (beam, target, recoil), there are twelve such observables in single-meson photoproduction. A so-called "complete" experiment, in which all complex amplitudes (apart from a single arbitrary phase) for the

\footnotetext{
ae-mail: Barry.Ritchie@ASU.edu
}

This is an Open Access article distributed under the terms of the Creative Commons Attribution License 4.0, which permits unrestricted use, distribution, and reproduction in any medium, provided the original work is properly cited. 


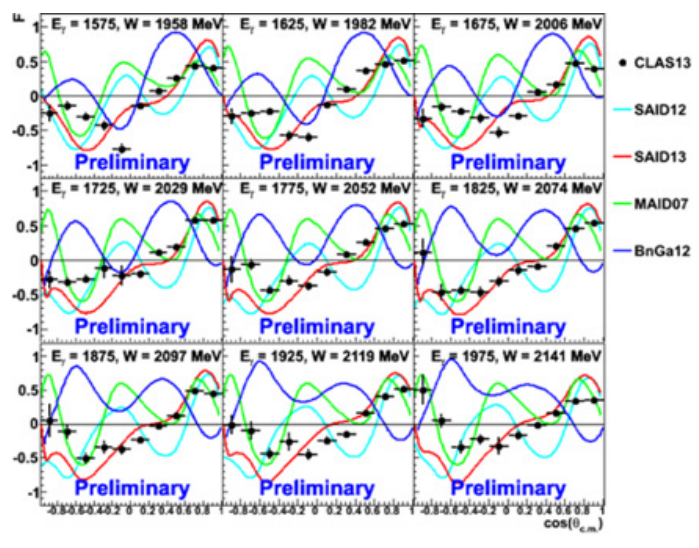

Figure 1. Preliminary results for $F$ for the reaction $\vec{\gamma}+\vec{p} \rightarrow n+\pi^{+}$using FROST. Curves shown are predictions from SAID [6], MAID 2007 [7], and Bonn-Gatchina [8].

helicities can be specified, is possible by measuring the differential cross sections, the beam and target asymmetries $\Sigma$ and $T$, and a judicious choice of the double-spin observables.

\section{The FROST program at Jefferson Lab}

A program of measurements for meson photoproduction at Jefferson Lab that used the photon tagger (capable of providing circularly and linearly polarized, as well as unpolarized, photon beams) [3] and the CEBAF Large Acceptance Spectrometer (CLAS) spectrometer in Hall B [4] has been extended by the development of a FROzen Spin Target (FROST) [5]. The hydrogen content in butanol $\left(\mathrm{C}_{4} \mathrm{H}_{9} \mathrm{OH}\right)$ beads, doped with the paramagnetic radical TEMPO, was used for meson production target protons in a cryogenic target. Using dynamic nuclear polarization, these target protons were oriented in a $5 \mathrm{~T}$ polarizing magnetic field at $0.3 \mathrm{~K}$ with microwaves of $\sim 140 \mathrm{GHz}$. At that point, the butanol material was cooled to $30 \mathrm{mK}$, after which the magnetic field was reduced to a holding magnetic field of $0.5 \mathrm{~T}$, such that most of the original polarization of the target nucleons in the stronger magnetic field was preserved. Both the cryogenic target and holding magnet were then inserted into CLAS.

Data obtained in running period "g9a" had the target proton polarization parallel or anti-parallel with respect to the incoming beam direction (i.e., longitudinal target polarization). Data obtained in running period " $\mathrm{g} 9 \mathrm{~b}$ " had the target proton polarization perpendicular to the incoming beam axis (i.e., transverse target polarization). Less than $10 \%$ of the time within these running periods was used to change polarization orientation. Within g9a and g9b were periods when the incident photons were either circularly or linearly polarized. Thus, running period g9a (longitudinally-polarized target) provided data for the single-meson photoproduction observable $E$ with circularly polarized photons and for $G$ with linearly polarized photons. Similarly, running period g9b (transversely-polarized target) provided data for the single-meson photoproduction observables $F$ and $T$ with circularly polarized photons and $H$ and $P$ with linearly polarized photons. (The recoil polarization $P$ can be derived from beam-target polarization measurements, or, as noted below in the case of recoil hyperons, directly measured.) Typical target nucleon polarizations during both $\mathrm{g} 9 \mathrm{a}$ and $\mathrm{g} 9 \mathrm{~b}$ were $\geq 80 \%$.

\section{Single-pion photoproduction}

Data were obtained for pion photoproduction on the proton in g9a for both $E$ and $G$, as well as $F, T$, $H$, and $P$ during $\mathrm{g} 9 \mathrm{~b}$. At present, ongoing analyses for $\pi^{+}$photoproduction have provided preliminary results for $T$ and $F$ from the Arizona State University (ASU) group, $E$ from the South Carolina (USC) group, and $G$ from the Edinburgh (UE) group; Figure 1 provides a sample of those results for $F$. For $\pi^{0}$ photoproduction, preliminary results for $E$ are also available from the ASU group. Taken together, 


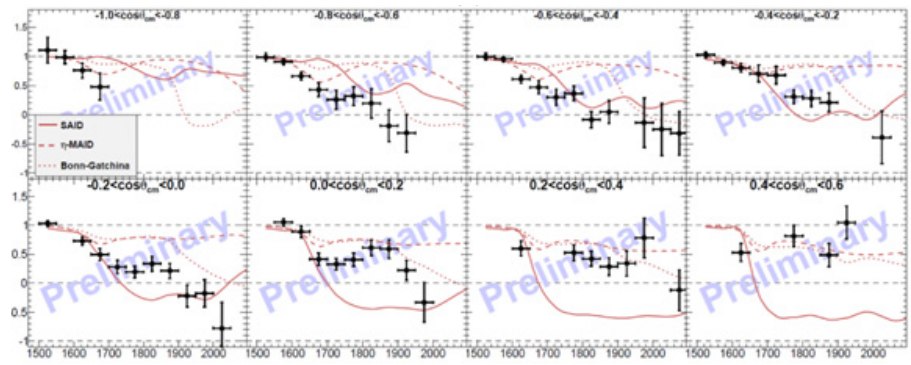

Figure 2. Preliminary results for $E$ for the reaction $\vec{\gamma}+\vec{p} \rightarrow p+$ $\eta$ as a function of center-of-mass energy $W$ using FROST. Curves shown are predictions from SAID [6], eta-MAID 2007 [9], and BonnGatchina [8].

these datasets are well-described by SAID [6], MAID 2007 [7], and Bonn-Gatchina [8]. at center-ofmass energies $W \leq 1.8 \mathrm{GeV}$, but those predictions are far less satisfactory at higher energies. Thus, even these very preliminary comparisons suggest much progress should come from theoretical efforts incorporating the final results of these studies.

\section{Single-eta and single-kaon photoproduction}

Studies of the $\eta$ and the $K \Lambda$ photoproduction reactions with polarized beam and target can take additional advantage of the "isospin filter" aspect of those reactions, selecting the isospin- $\frac{1}{2}$ resonances. In the preliminary analyses underway, observations of $E$ (from g9a) and $F$ and $T$ from g9b have become available for $\eta$ photoproduction from the ASU group, and g9b studies have also yielded $F$ and $T$ results for kaon photoproduction from the Catholic University group. An example of some of those preliminary results for the $E$ observable in $\eta$ photoproduction is shown in Fig. 2, where it is seen that the SAID [6], $\eta$-MAID [9], and Bonn-Gatchina [8] predictions are generally inconsistent with the preliminary results at all energies for forward angles where $t$-channel effects are usually greatest.

In kaon photoproduction, the weak decay of the hyperon allows extraction of the hyperon polarization, permitting a determination of the recoil hyperon polarization. This gives an additional double-spin observable that can further illuminate the resonance spectrum populated by that reaction. The early FROST analyses, focusing on the simpler observables, have not exploited this feature yet, but this will likely be very useful in pinning down the helicity amplitudes for hyperon final states.

\section{Multi-pion photoproduction}

Large-solid-angle detectors are excellent tools to study reactions where more than one meson appears in the final state. Such reactions can provide a new avenue to investigate nucleon structure and help identify resonances that decay into other resonances. The spin combinatorics for the two-pion final states can appear daunting, however: 64 observables, 28 independent relations related to helicity amplitude magnitudes, 21 independent relations related to helicity amplitude phases, resulting in 15 independent numbers needed to describe the process $[10,11]$.

Preliminary studies of the $P_{z}$ observable (for definitions, see, e.g., Refs. [10, 11]) for the reaction $\vec{\gamma}+\vec{p} \rightarrow p+\pi^{-}+\pi^{+}$have been carried out by the Florida State (FSU) and USC groups using the g9a dataset. Additionally, preliminary results for the $P_{z}^{s}$ and $P_{z}^{c}$ observables have been obtained through analyses by USC, and for $P_{z}^{\circ}$ by FSU; results for other observables can also be expected in the future from both g9a and g9b runs. One set of preliminary results from g9a studies is shown in Fig. 3, compared with predictions by Roberts and Oed [10] and by Fix and Arenhövel [11]. 


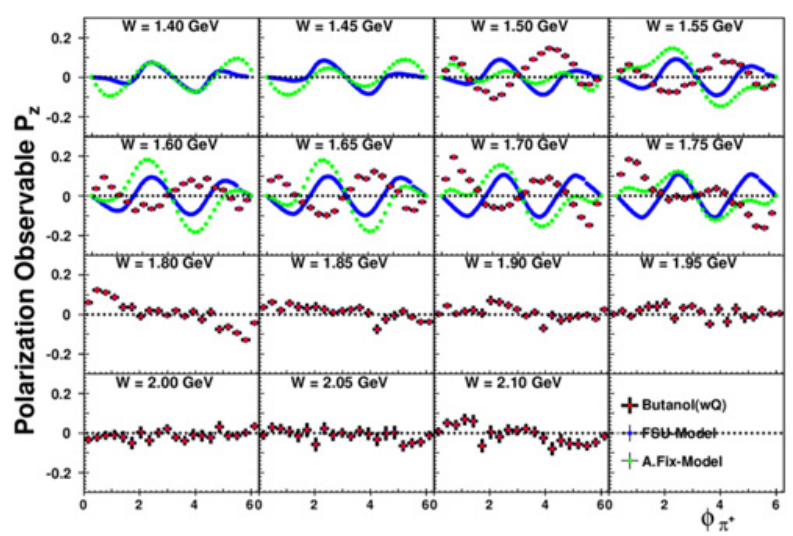

Figure 3. Preliminary results for $P_{z}$ for the reaction $\vec{\gamma}+\vec{p} \rightarrow p+\pi^{-}+\pi^{+}$as a function of the out-of-plane angle $\phi_{\pi^{+}}^{*}$ for various center-of-mass energies $W$ using FROST. Curves shown are predictions for the process from predictions by Roberts and Oed (blue) [10] and by Fix and Arenhövel (green) [11].

\section{Conclusion}

The polarized-photon/polarized-target experiments in Hall-B with FROST at JLab have acquired hundreds of data points yielding clues to the nucleon resonance spectrum. For most channels, data will be sufficient for a nearly complete experiment. For $K \Lambda$ and $K \Sigma$ channels, a complete experiment may be feasible. Double-pion observables offer a "next generation" probe of reaction mechanisms to glimpse resonances not clearly seen in single-meson photoproduction. Just the small subset of FROST preliminary results discussed above already confirms that spin observables will tremendously aid sleuthing out resonance parameters and finding missing resonances (if they exist). Data from analyses of several reactions are nearing the publication stage, but considerable work remains yet to be done.

The preliminary results shown in this overview of FROST studies came from individual analyses being conducted by M. Dugger (ASU), S. Park (FSU), I. Senderovich (ASU), and N. Walford (CUA); additional results shown in Rome (but not shown here due to space limitations) came from J. McAndrew (UE), Y. Mao (USC), S. Strauch (USC), and R. Tucker (ASU).

Work at ASU is supported by a grant from the U. S. National Science Foundation.

\section{References}

[1] J. Beringer, et al. (Particle Data Group), Phys. Rev. D 86, 010001 (2012)

[2] I.S. Barker, A. Donnachie, and J.K. Storrow, Nucl. Phys. B 95, 347 (1975)

[3] J. O'Brien, et al. (CLAS Collaboration), Nucl. Instrum. Meth. Phys. Res. A 440/2, 263 (2000)

[4] B. Mecking, et al. (CLAS Collaboration), Nucl. Instrum. Meth. Phys. Res. A 503, 513 (2003)

[5] C.D. Keith, et al., Nucl. Instrum. Meth. Phys. Res. A 684, 27 (2012)

[6] R.L.Workman, M.W. Paris,W.J. Briscoe, and I.I. Strakovsky, Phys. Rev. C 86, 015202 (2012)

[7] The MAID analyses are available at http://wwwkph.kph.uni-mainz.de/MAID/. See also D. Drechsel, S.S. Kamalov, and L. Tiator, Eur. Phys. J. A 34, 69 (2007)

[8] A.V. Anisovich, E. Klempt, V.A. Nikonov, A.V. Sarantsev, and U. Thoma, Eur. Phys. J. A 47, 153 (2011)

[9] W.-T. Chiang, S.-N. Yang, L. Tiator, and D. Drechsel, Nucl. Phys. A 700 429-453 (2002)

[10] W. Roberts, T. Oed, Phys. Rev. C 71, 055201 (2005)

[11] A. Fix and H. Arenhövel, Phys. Rev. C 87, 045503 (2013) and to be published 